\title{
Robots for Learning
}

\author{
Wafa Johal $^{1}$ (D) - Ginevra Castellano ${ }^{2} \cdot$ Fumihide Tanaka $^{3} \cdot$ Sandra Okita $^{4}$
}

Published online: 7 June 2018

(c) Springer Science+Business Media B.V., part of Springer Nature 2018

\section{Motivation}

Using robots for education is not something unheard of. Papert with the LOGO turtles was one of the pioneers in the early 1980's introducing robots in classrooms. Since then, robots have been used more and more used to teach programming and other STEM related fields. Supported by governmental decisions that have been taken around the world to train students earlier to technologies and engineering earlier, the breakthrough of robots in everyday teaching practice is not yet visible. This resistance to the acceptance of robots in educational contexts can be explained by several factors among which are the cost of robots, their limited application in curricular activities, and the lack of training of teachers and their inclusion in the robot's design.

However, since the avent of the 4th industrial revolution and the recent major advances in robotics, artificial intelligence and computer sciences, robots have become more reliable, cheaper and more acceptable. These robots with new and improved capabilities bring new potential for social inter-

\footnotetext{
${ }^{1}$ https://r4l.epfl.ch.

Wafa Johal

wafa.johal@epfl.ch

Ginevra Castellano

ginevra.castellano@it.uu.se

Fumihide Tanaka

tanaka@iit.tsukuba.ac.jp

Sandra Okita

Okita@tc.columbia.edu

1 CHILI/ LSRO Labs, École polytechnique fédérale de Lausanne, EPFL IC IINFCOM CHILI RLC D1 740 (Rolex Learning Center) Station 20, 1015 Lausanne, Switzerland

2 Department of Information Technology, Uppsala University, Lägerhyddsvägen 2, 75105 Uppsala, Sweden

3 Faculty of Engineering, Information and Systems, University of Tsukuba, Tennodai 1-1-1, Tsukuba, Ibaraki 305-8573, Japan

4 Department of Mathematics, Science and Technology (Box 8), Teachers College - Columbia University, 525 West 120th Street, New York, NY 10027, USA
}

action with learners. Being able to sense, they could provide adapted feedback to learners and monitor the classroom for the teachers. In comparison with computer assisted learning, robots will have the advantages of providing a multi-modal embodied learning experience.

After a series of three workshops on the topic of Robots for Learning, ${ }^{1}$ this special issue seeks to offer interdisciplinary perspectives on the future of child-robot interaction in educational contexts. The goal is to foster discussions and investigations in social robotics, to be able to provide successful interactions. Several aspects and perspectives are taken by the authors. From STEM to second language, the authors have explored various curricular activities. With animal-like robots, humanoids or robotic kits, we observe an heterogeneity in their design. Finally, the impact of the use of these robots in learning activities shows how complex it is to design a tool for classrooms or educational contexts.

\section{Content}

The special issue consists of six high quality research papers.

In "How could companion robots be useful in rural schools?", Elizabeth Broadbent, Danielle Alexis Feerst, Seung Ho Lee, Hayley Robinson, Jordi Albo-Canals, Ho Seok Ahn and Bruce A. MacDonald, describe a crosssectorial study in rural area exploring views of 207 students and 22 teachers on the use of two robots in educational settings. They introduced a Paro and a IRobovi robot during 30-min group sessions with a demo and open-ended questions. They authors specifically asked teachers about the tasks the robot could perform at school and how useful it could be. This study contributes towards the definition of the role of the companion robots in schools. The results also underline the gender difference and potential new tasks for robots in rural sector schools.

"Between the Social and the Technical: Negotiation of Human-Centered Robotics Design in a Middle School Classroom" by Andrea Gomoll, Selma Sabanovic, Erin Toal, Cindy E. Hmelo-Silver, Mattew R. Francisco and Orion Lawlor, reports on a human-centered robotics learning expe- 
rience. Willing to increase the engagement of students in STEM practices, the authors designed a project in which students could build a robot relevant for daily lives and societal issues. Through several examples of students outcomes, authors show that challenges and successes in engaging students with STEM rely on both coal and technical questions and skills.

The pedagogical and child-robot interaction issues are the focus of the article "Guidelines for designing social robots as second language tutors" by Tony Belpaeme, Paul Vogt, Rianne van den Berghe, Kirsten Bergmann, Tilbe Gsun, Mirjam de Haas, Junko Kanero, James Kennedy, Aylin C Kntay, Ora Oudgenoeg-Paz, Fotios Papadopoulos, Thorsten Schodde, Josje Verhagen, Christopher D Wallbridge, Bram Willemsen, Jan de Wit, Vasfiye Gekin, Laura Hoffmann, Stefan Kopp, Emiel Krahmer, Ezgi Mamus, Jean-Marc Montanier, Cansu Oran and Amit K Pandey. The authors investigate the challenges in the view of a second language robot tutor. They raise several issues and propose a series of guidelines to design the tutoring robot. After introducing in second language learning and social robot tutoring, the authors expose several design issues related to the pedagogical aspects of the tasks or child-robot interaction. Supported by empirical evidences, the authors propose a number of practical solutions in the use of robots for second language tutoring.

Attention, a crucial component of in learning interaction is the core investigation of the next paper. In "Directing Attention through Gaze Hints Improves Task Solving in Human-Humanoid Interaction" by Eunice Njeri Mwangi, Emilia I. Barakova, Marta Daz - Boladeras, Andreu Catal Mallofr and Matthias Rauterberg, authors report an experimental study designed to examine how participants interpret and perceive social hints from gaze exhibited by either a robot or a human tutor when carrying out a matching task. They propose several conditions to test if the gaze behavior of the robot would influence the participants' performances in a card matching memory game. Their findings show that the robot tutor was efficient in giving gaze hints (making the task easier). Even better these gaze cues were better recognized comparatively to human tutor gaze hints. This study provides a good example of how nonverbal cues of communications can be manipulated to improve learning performances.

An open-leaner model and a concrete implementation of long-term interactions with a robotic tutor are proposed by the authors of the next article. In "Adaptive robotic tutors that support self-regulated learning: A longer-term investigation with primary school children" by Aidan Jones and Ginevra Castellano, the authors propose to use a robotic tutor to promote self regulated learning skills. This paper explores how personalized tutoring by a robot achieved using an open learner model (OLM) promotes SRL processes and how this can impact learning and SRL skills compared to personalized domain support alone. Authors present a long-term study where participants take part in a geography-based task on a touch screen with adaptive feedback provided by the robot. In addition to domain support the robotic tutor uses an OLM to prompt the learner to monitor their developing skills, set goals, and use appropriate tools. The authors findings show that personalization and adaptivity scaffold SRL behavior based upon an OLM.

Development of children with special needs is at the center of Jordi Albo Canals, Marina Umaschi Bers, Alex Barco, Daniel Hannon, Marcel Heerink, Martina Heinemannd, Kaitlyn Leidl and Emily Relkin "A Pilot Study of the KIBO Robot in Children with Severe ASD". In this paper, the authors explore how KIBO a programmable toy robot could be used to teach coding and sequencing to children with ASD. In their study with 12 children, they report qualitative and quantitative analysis of the interactions. Although a significant learning was not observed, authors present evidences showing a sustained interest in the KIBO robot and an increased the frequency of their interactions with adults across play sessions.

The guest editors hope that the articles featured in this special issue will inspire further research in various fields of social robotics and education and foster productive interdisciplinary research findings. We wish to thank all of the contributors, reviewers and editors without whom the realization of this special issue wouldn't have been possible.

Acknowledgements Authors would like to thank supporters of the series of workshop on Robots for Learning : Swiss National Science Foundation through the National Centre of Competence in Research Robotics, the EU Project ANIMATAS (MSCA - ITN - 2017 - 765955 2), and KAKENHI (15H01708).

Publisher's Note Springer Nature remains neutral with regard to jurisdictional claims in published maps and institutional affiliations.

Wafa Johal obtained her PhD in 2015 from the University of Grenoble, France focusing on body signal communication in Child-Robot Interaction. She is a postdoctoral researcher in the Computer and Human Interaction for Learning and Instruction laboratory at EPFL, Switzerland. She works within the CoWriter, Cellulo and Animatas projects dealing with designing human-robots interaction for education.

Ginevra Castellano is an senior lecturer in intelligent interactive systems at the Department of Information Technology of Uppsala University, where she leads the Uppsala Social Robotics Lab. She was the coordinator of the EMOTE project (2012-2016), which developed educational robots to support teachers in a classroom environment. She is currently Principal Investigator of several national and Europeanfunded projects on educational robots.

Dr. Fumihide Tanaka is an Associate Professor of Department of Intelligent Interaction Technologies in University of Tsukuba. He works for Social Robotics and Human-Robot Interaction.

Sandra Okita is an Associate Professor of Technology and Education at Teachers College, Columbia University. Her current research interest is focused on designing a learning partnership between individuals and technology (e.g., robots, pedagogical agents) in STEM education, and how technology intersects with learning and instructional processes. 